\title{
Surveillance Chest X-Rays for Melanoma: Prescription or Proscription?
}

\author{
Sandra L. Wong, MD, MS \\ Division of Surgical Oncology, Department of Surgery, University of Michigan, Ann Arbor, MI 48109-0932
}

The presence of metastases in the regional nodal basin is the most powerful prognostic indicator for patients with melanoma. Long-term postoperative surveillance is important for these high-risk patients, but there remain questions about the appropriate frequency and intensity of follow-up. Routine clinical follow-up allows for detection of recurrences or offers assurance to patients that they are cancerfree. In theory, earlier detection of disease has the laudable goal of allowing for earlier (and more effective) treatments. No controversy would exist if clinical examination or imaging tests were sensitive and specific for the detection of conditions that, if early enough in their course, could be treated successfully. However, there are data to suggest that most patients with recurrent melanoma were symptomatic at the time of diagnosis even with regularly scheduled followup visits, and often, treatment options are limited. ${ }^{1}$

For high-risk melanoma patients, such as those with micrometastatic nodal disease, one of the most common sites of distant metastases is the lungs. Although screening with blood work and chest X-rays (CXR) have been shown to have low utility for detection of pulmonary metastases, they are routinely obtained as part of postoperative surveillance plans. ${ }^{2}$ Current recommendations for CXR, or other imaging modalities, in this patient population are nonspecific and are largely based on consensus opinion. For example, the National Comprehensive Cancer Network guidelines include CXR every 6-12 months for 2-3 years, although it acknowledges the absence of clear data for such recommendations. ${ }^{3}$

In this issue of Annals of Surgical Oncology, Morton et al. report on their prospective experience with surveillance

(C) Society of Surgical Oncology 2008

First Received: 25 October 2008;

Published Online: 18 December 2008

S. L. Wong, MD, MS

e-mail: wongsl@umich.edu
CXR in patients with microscopically node-positive melanoma. ${ }^{4}$ In this study, 23 of 108 patients ultimately had findings of pulmonary metastases over a 52.5-month median follow-up period. Earlier detection of metastases may be beneficial because asymptomatic patients appeared to do better than symptomatic patients. However, $<50 \%$ of patients with pulmonary metastases were found to have disease on surveillance CXR, indicating its relative uselessness. Furthermore, there was a relatively high falsepositive rate, leading to patient anxiety and further workup that may not be without cost and morbidity.

It seems clear from this study that CXR is neither sensitive nor specific enough to serve as an effective surveillance examination for melanoma patients. This study has several limitations to consider. In the analysis, $>70 \%$ of sentinel node-positive patients were excluded because they did not have serial CXR as part of their routine follow-up. The study population may represent lower-risk patients because the higher-risk patients may have been found to have metastatic disease after initial sentinel node biopsy or were deemed high risk enough to undergo more sensitive imaging modalities such as computed tomography, magnetic resonance imaging, or positron emission tomography as a part of their routine follow-up. It may be interesting to know how many patients initially staged with CXR went on to other imaging modalities at undefined intervals. Because only $13 \%$ of patients with CXR-detected pulmonary metastases underwent metastasectomy, it may be important to know if surveillance chest computed tomographic scans would lead to meaningful earlier detection or if they would just confound follow-up because they are too sensitive.

Most recurrences after resection of primary melanomas are discovered by clinical evaluation, including a thorough history and physical examination. It may seem harmless to recommend a low-cost, minimally invasive test, but the data from Morton et al. are proscriptive. CXR are not helpful in the surveillance of melanoma patients, and there 
is no role for this test in patients with micrometastatic disease in their sentinel nodes. Guidelines that continue to recommend or allow for this type of examination should be reconsidered, and efforts should be redirected toward more effective and efficient surveillance modalities.

\section{REFERENCES}

1. Shumate CR, Urist MM, Maddox WA. Melanoma recurrence surveillance: Patient or physician based? Ann Surg 1995;221: $566-71$.
2. Weiss M, Loprinzi CL, Creagan ET, et al. Utility of follow-up tests for detecting recurrent disease in patients with malignant melanomas. JAMA. 1995;274:1703-5.

3. NCCN Clinical Practice Guidelines in Oncology. Version 1.2009. Available at: http://www.nccn.org/professionals/physician_gls/ PDF/melanoma.pdf. Accessed November 18, 2008.

4. Morton RL, Craig JC, Thompson JF. The role of surveillance chest $\mathrm{X}$-rays in the follow-up of high-risk melanoma patients. Ann Surg Oncol. DOI:10.1245/s10434-008-0207-5. 